\title{
Switch techniques to recover spatial consistency between virtual and real world for navigation with teleportation
}

\author{
Yiran Zhang ${ }^{1}$, Nicolas Ladevèze ${ }^{1}$, Cédric Fleury ${ }^{2}$, and Patrick Bourdot ${ }^{1}$ \\ 1 VENISE Team, LIMSI, CNRS, Univ. Paris-Sud, Université Paris-Saclay, \\ Orsay, France \\ 2 Univ. Paris-Sud, CNRS, Inria, Université Paris-Saclay, Orsay, France
}

\begin{abstract}
In many virtual reality systems, user physical workspace is superposed with a particular area in a virtual environment. The spatial consistency between the real and virtual interactive space allows users to take advantage of physical workspace to walk and to interact intuitively with the real and virtual contents. To maintain such spatial consistency, application designers usually deactivate user virtual navigation capability. This limits user reachable virtual area, and segments the spatial consistency required sub-task from a continuous scenario mixing large scale navigation. In order to provide users with a continuous virtual experience, we introduce two switch techniques to help users to recover the spatial consistency in some predefined virtual areas with teleportation navigation: simple switch and improved switch. We conducted a user study with a box-opening task in a CAVE-like system to evaluate the performance and usability of these techniques under different conditions. The results highlight that assisting the user on switching back to a spatially consistent situation ensures entire workspace accessibility and decreases time and cognitive effort used to complete the sub-task. The simple switch results in less task completion time, less cognitive load, and is globally preferred by users. With additional visual feedback of user switch destination, the improved switch seems to provide the user with a better understanding of the resulting spatial configuration of the switch.
\end{abstract}

Keywords: Virtual reality $\cdot$ 3D interaction $\cdot$ Teleportation.

\section{Introduction}

In virtual reality systems, user physical interaction space, also named physical workspace, is the area of the real world where users can interact with a virtual environment. This area is usually constrained by the available motion tracking

This is the author's version of the work. It is posted here for your personal use. Not for redistribution. The definitive version of record was published in Lecture Notes in Computer Science(Springer), https://doi.org/10.1007/978-3-030-31908-3_1 
area, restricted by the presence of physical obstacles inside this area and the range of devices (e.g. haptic). The physical interaction space merges all those restrictions and defines the capability of the virtual reality (VR) setup. The corresponding virtual interaction space is an area in the virtual environment dedicated to virtual task performance, named "stage" [11] or "vehicle" [7] in some prior studies. The spatial consistency between the physical and the virtual interaction spaces is usually maintained to avoid the usage of navigation metaphors in the virtual interactive spaces where successive interactions are involved. In a one-to-one scale, user real and virtual interaction spaces can be completely consistent by possessing identical form, coordinate system, and scale.

Spatial consistency allows the user to use the maximum physical workspace to interact with a virtual environment. Users can thus get access to the required virtual objects by walking, and interact intuitively inside the virtual environment without colliding with real obstacles. Spatial consistency also allows some tangible interaction designs. For example, using a physical object to represent objects relevant to the virtual content is widely used in many virtual reality experiences, named substitutional reality in some prior studies [21]. The use of a physical object provides users with passive haptics feedback and increases user sensation of presence [15]. In a multi-user context, the spatial consistency allows users being presented in a virtual environment following their real spatial configurations, which enables offering users a sharing tangible interface (e.g. props) to coordinate their movements during virtual co-manipulation $[18,22,19$, $1,9]$. Moreover, spatial consistency enables real user and avatar superimposition, which enhances social awareness in multi-user situations.

User navigation capabilities are usually constrained for spatial consistency maintenance, which limits user reachable area in the virtual scene. For example, in a virtual windshield assembly task, users were asked "walk about 2 meters" [14] and the virtual interaction space for a virtual car hood assembly task was restricted as $4 \mathrm{~m} \times 4 \mathrm{~m}$ [1]. The main issue for limiting navigation capabilities is that it breaks user sensation of free exploration and segments tasks that are continuous in real scenarios.

Virtual navigation allows users exploring the virtual environment beyond the physical workspace limitation. However, when individual navigation capability is provided, what a user perceives in the virtual environment may no longer be consistent with its spatial distribution in the real world. Complex scenarios that include free navigation is incompatible with consistency maintenance. In order to provide users with a continuous virtual experience, a seamless switch is necessary to help users recover spatial consistency after a free virtual navigation phase. This paper focuses on the design and evaluation of such switch techniques.

Introducing such switch techniques enriches the possible scenarios in both single and multiple user contexts. With the switch, users can navigate between specific areas of interest defined in the virtual environment and can achieve successively some tasks that require spatial consistency. A possible scenario can be an escape game where a user is required to escape from imprisonment by exploiting a series of virtual rooms. The switch can help the user to recover the 
spatial consistency in some specific area where the clues are hidden. Hence, the user can walk, explore the virtual surrounding and manipulate with the virtual contents in this area with full accessibility. After that, the user can navigate to another room to continue the adventure. Another example can be a virtual assembly training. Users firstly navigate individually to find the required mechanical pieces stocked in different virtual warehouses. The spatial consistency will be recovered when users arrive at a specific area where mechanical pieces are assembled, and the user movement can be coordinated with a sharing prop.

The switch techniques should adapt to the navigation metaphor to maintain a continuous sensation for the whole virtual experience. In this paper, we investigated two switch techniques for teleportation navigation: simple switch and improved switch. The simple switch allows the user quickly recovering the spatial consistency with two steps: select and activate. Since having correct anticipation of the resulting spatial configuration is helpful for users to perform the following task, additional visual feedback of user switch destination is provided in the improved switch approach. We conducted a user study to compare the two switch techniques with a baseline (free navigation without switch) in a box-opening task. First, we want to confirm weather helping users to recover spatial consistency will increase sub-task performance compared to complete unrestrained navigation. Then, we want to know if the improved switch brings add values compared to the simple switch.

In Sect.2, we first propose a classification of the existing applications based on spatial relationships between the real and virtual interaction space. Then we review the prior works relate to spatial consistency and teleportation navigation. In Sect.3, we describe our two switch approaches for teleportation navigation. Then in Sect.4, we describe our experiment design, derive the hypotheses and present the evaluation and the results. Finally, we critically discuss these results in Sect. 5 and conclude in Sect.6.

\section{Related work}

In many virtual reality applications, the user physical workspace is consistent with a specific interaction space defined in the virtual environment according to target scenario. In this section, we firstly propose a classification of the existing applications based on the coupling mechanism of real and virtual interaction space. Secondly, we detail the interests of spatial consistency by presenting the existing virtual reality application designs. Thirdly, we highlight the previous work which attempted to maintain the spatial consistency during navigation. Finally, as we choose to experiment our switch techniques on a specific navigation metaphor, namely teleportation, we review navigation techniques based on this metaphor and discuss their limitations.

\subsection{Spatial consistency}

Depending on how real and virtual interaction space is coupled, the spatial relation between real and virtual world can be varied from a complete to a partial 
consistency. In complete consistency situation, user's real and virtual interaction spaces are fully superposed and possess identical form, coordinate system, and scale $[18,1]$. The spatial relation can also be partially consistent when user real interaction space intersects with the real interaction space. For example, in a distributed collaborative system, users usually possess different real workspace configurations and immerse in the same virtual environment. By overlapping a part of each user's real interaction space to the shared virtual interaction space, users can perform a collaborative task inside the overlapping area. Simultaneously, each user retains a certain part of the interaction area to perform some individual tasks. In some cases, users are no longer located in the same virtual environment, but the spatial relation among users maintains consistent and is used for application designs. For example, in Mutual Turk system [9], users exchange force via a sharing prop. Their timelines are synchronized so that the way for manipulating the shared prop is consistent across different virtual worlds: one user pilot the kite while another user tugs fish out of pounds.

The spatial consistency between the real and virtual workspace allows users to take advantage of physical workspace to walk and interact intuitively with the virtual surroundings. Real walking is the most direct and natural technique for traveling in the virtual environment. It promotes spatial understanding and provides users with vestibular cues which help them understand the size of the environment [3]. Compared to other alternative locomotion techniques, walking offers a higher immersive experience in virtual reality applications [26].

The spatial consistency also enables the tangible interaction design in many virtual reality applications. For example, CavePainting [16] allows the artist to takes advantage of $8 \mathrm{ft} . \times 8 \mathrm{ft} . \times 8 \mathrm{ft}$. space to create a new type of art using props and gestures. The iTurk system [8]complements virtual reality experiences with passive props. The main idea behind this system is using user to reconfigure and animate otherwise passive prop in the virtual reality. Also, in substitutional reality systems, physical object and the architectural feature can be incorporated into the virtual world by paring them to a virtual counterpart [24]. For example, in the Simeone et al. study, a living room can be replaced by a courtyard and an umbrella can be replaced by a sword or a lightsaber [21]. The real-world object provides tangibility to the paired virtual object and contributes to a higher sense of presence [15].

The spatial consistency is also necessary for multi-user system design. It simplifies the spatial information communication and enables the use of passive haptics feedback for co-located users. In projection based system, co-located users can communicate the coordinate information and negotiate common goals implicitly using social cues like gesture, facial expression and gazing, etc. User can point [22] or draw the outline of an object [19] directly in the air to illustrate his desired object to the collaborator. With several head-mounted displays, multiple tracked users can work on medical training side-by-side [20]. Moreover, spatial consistency enables the possibility of offering sharing tangible interfaces for co-located users. A tangible prop can be designed to coordinate the user 
movement for co-manipulation of the virtual windshield [18] and the virtual car hood [1].

In the above designs, to maintain spatial consistency, user reachable virtual workspace is usually constrained/limited. To reduce the severity of physical workspace restrictions, Suma et al. [23] proposed to compress relatively large interior environments into smaller overlapping areas. The geometry of a new area will replace the previous one when users reach a transition area. User can thus naturally walk through these sub-areas where the spatial consistency is maintained. Other approaches like virtual navigation techniques can also be deployed to break the restrictions imposed by the limited physical interaction space. However, when the user is provided with individual navigation capability, the virtual spatial configuration will diverge from the real-world situation. Some prior studies tried to maintain spatial consistency during virtual navigation. For a single user, the 3DM allows the user to walk on a "magic carpet" which marks the boundaries of the tracking system. For long-distance navigation, the magic carpet can be translated following user pointing direction [6]. Magic Barrier Tape allows the user to walk inside the physical workspace surrounded by a virtual barrier tape. User can move beyond the boundaries by "pushing" the tape [10]. For multiple users, Multi-Ray jumping [25] allows group navigation while the spatial consistency among co-located users is maintained. Intensive interactions usually occur and concentrate in certain areas of the virtual environment. However, these metaphors cannot guarantee users getting access to all the required virtual objects inside such areas without using virtual navigation techniques. Consequently, the user needs to maintain the accessibility of workspace using virtual navigation metaphor and performs the associated sub-task at the same time, which may increase the cognitive load and the time used for completing the task.

In this paper, we propose approaches to help the user to recover the spatial consistency in such an area. Users can thus get access to all needed virtual objects by walking and focus on the sub-task performance without using virtual navigation metaphor. As these specific areas are integrated into the virtual environment according to the target scenario, users can successively explore these specific areas following a story-line. By overlapping multiple users physical workspace to a shared virtual interactive space, our approach can also be easily extended to meet the demands of multiple user scenarios.

\subsection{Teleportation navigation}

The concept of switch can be applied to many navigation metaphors. In this paper, we focus on teleportation navigation since it is widely used in many VR applications and VR games. Some prior studies shows that teleportation is able to reduce motion sickness [27]. Since no visible translation motion is involved during navigation, it avoids the sensory conflict between the visual and vestibular system of a user [17]. Although teleportation offers less spatial information for path integration, the presence and the spatial orientation that users perceived did not seem to be affected [27]. 
With teleportation technique, user viewpoint will be instantly transferred to a target position defined by the user. This process can be generally divided into two stages: the selection of the target destination and the activation of teleportation. In most applications, the target teleportation position is specified using a tracked input device [5]. In some cases, this process is implemented without the involvement of control variables. For example, the destination can be determined by extending user viewing direction from the virtual head position [2] or asking user walking directly into a virtual portal [12]. Most teleportation metaphors are limited to two dimensions, user future orientation can only be corrected by physical movement after the teleportation $[2,4]$, which may not be suitable for some CAVE-like system due to the missing screen. To perform teleportation with three degrees of freedom (3dof), i.e. setting both a 2D position and an orientation on a plane (1D rotation), we followed the same mechanism design proposed by Bozgeyikli et al. [5] and replaced the direct hand gesture mapping by the linear gain function mapping for the ergonomic reason.

Teleportation can be activated without an additional input device. For example, teleportation will be activated when users point to the same place or a close vicinity for two seconds [5] or with a jumping movement [30]. However, these approaches may make the user tired and impatient during long-term navigation. Our approach followed the common design and the teleportation is activated by pulling the trigger on an input device.

Blur effect [2] and fade-in and fade-out effect [12] are proposed for helping user adapt to the instant change of the virtual world. However, the former may induce motion sickness, and the later may break immersion and prolong the time for each teleportation. Thus, in our approach, the user's virtual viewpoint will instantaneously move to the pointed destination once the teleportation is triggered.

\section{Switch techniques}

In our implementation, application designers can define some specific areas (denoting interactive area below) in the virtual environment where successive interactions are involved. These interactive areas cover the required virtual interaction spaces for completing a sub-task and follow the same layout of the user real interaction space. After the user enters an interactive area, spatial consistency between user real and virtual interaction space can be recovered by superposing user real interaction space with the interactive area. The transition from free navigation to spatial consistency situation allows the user to walk inside the interactive area and interact intuitively with the virtual soundings to complete the corresponding task. By strictly superposing all users' physical workspace to the same virtual interactive area, this approach is also applicable in a multi-user context.

Two switch techniques are provided for users to select their desirable interactive area and perform such transition:simple switch and improved switch (Fig. 1). 


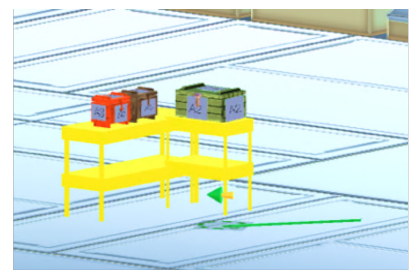

(A)

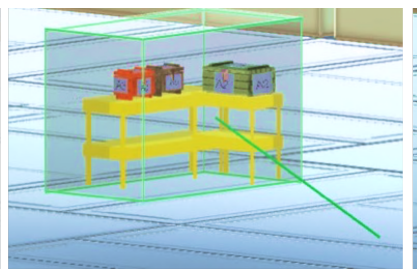

(B)

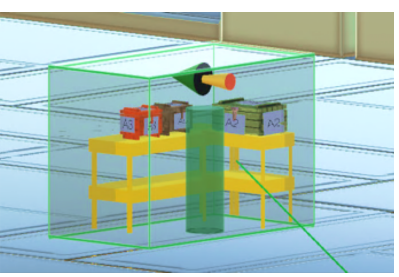

(C)

Fig. 1. (A) Without the switch. (B) Simple switch: the interactive area is highlighted when it is selected. (C) Improved switch: when the user selects an interactive area, it will be highlighted, and the user future position and orientation will be presented by a $3 \mathrm{D}$ arrow with a semi-transparent cylinder.

\subsection{Simple switch}

In this approach, the interactive area is presented as an invisible cube and has the same layout with the virtual presentation of the physical workspace of the user. The collision detection is performed between the cube and the laser beam used for teleportation. Users can select an interact area by pointing the laser beam to it. Once a collision is detected, the interactive area will be selected and presented as a transparent cube with a green border.

Users can activate the switch by pressing the same button used for activating teleportation. After that, users will be teleported into the selected interactive area. The position and orientation inside the interactive area (denoting switch destination below) are computed based on user's real position and orientation. Using teleportation inside the interactive area is invalid. The laser beam will present as red when users try to select a teleportation destination within the interactive area. Under such circumstances users will stay in place even the teleportation is triggered. Once users finish the task in the interactive area, they can continue the navigation by selecting a teleportation destination outside the interactive area. Thus, users can seamlessly switch back to the spatial inconsistent situation.

The main advantage of this technique is that the switch can be achieved very quickly and straightforwardly. However, users are teleported directly into the interactive area without any visual feedback of their future destination. After being teleported to a previously unknown environment, users need to capture the spatial information around and adapt to the new environment by reconstructing a virtual representation of surroundings in their mind. The lack of awareness of switch destination may prolong this procedure and make users disoriented after the switch.

\subsection{Improved switch}

Different than simple switch, the improved switch provides users with an additional visual feedback of their switch destination (Fig. 1). This visual feedback 
will be displayed when an interactive area is selected. Its position and orientation are computed by mapping user real-time position and rotation inside the physical interaction space to the corresponding interactive area.

Users can use this visual feedback to anticipate their future destination and locate themselves within a cognitive map of the interactive area. Therefore, before teleporting into the interactive area, users can glance at the target virtual object location relative to their future position and orientation, which is conducive for performing the following task. For example, by seeing the target object located on the right side of their switch destination, users can realize that after jumping into the zone, they can find the target object on their right side. Besides, users can move inside the physical workspace to slightly tune the switch destination while pointing to an interactive area. By observing the change of the visual feedback, users can have a better comprehension of the spatial relationship between the target object and their future destination. This may enhance their understanding of the resulting spatial configuration and improve their estimation.

The visual feedback of the switch destination should provide users with clear position and orientation information to ensure their anticipation. In our implementation, the visual feedback design extends the one used for teleportation. It consists of a semi-transparent cylinder with $0.5 \mathrm{~m}$ diameter and a $3 \mathrm{~d}$ textured arrow placed above the cylinder. Since the flat dotted circle used for teleportation can be easily blocked by other virtual objects near the interactive area, we replaced it by a cylinder for presenting the position of switch destination.

Using a ghost avatar to present the switch destination may increase user sensation of presence, help users getting self-related information and deepen their understanding of the relationship between the switch destination and their physical movement. Thus, before designing the experiment, other different types of switch destination visual feedback, including symbolic T-shaped like avatar and simple humanoid avatar, were tested in-house. However, these avatars can not offer users clear orientation information. Users need to infer its direction using other information such as the texture. Therefore, to ensure that users can get more accurate orientation information, we used the 3D textured arrow to present the orientation after the switch.

\section{User study}

In our experiment, we compared two switch conditions (simple switch and improved switch) with a third condition that offered no switch as a baseline (without switch). The objective of the experiment is to accomplish a box-opening task in the virtual environment.

\subsection{Experimental setup}

The experiment was carried out within a large CAVE-like system using the Unity game engine. Stereoscopic images are projected on all three screens surrounding 
a floor screen. User's motion is tracked by a tracking system composed of infrared cameras. User's head position and gaze direction are computed from the markers attached on shutter glasses. Thus, the adaptive stereoscopic images following the user viewpoint can be correctly rendered on the screen. User's hand information is computed from the marker installed on a Nintendo Wii remote controller held by the user dominant hand. This information will be used for ray casting and virtual manipulation task.

\subsection{Teleportation with a specific direction}

The teleportation destination is determined in the virtual environment by ray casting. For reducing the discomfort from wearing a redundant tracking device, in our implementation, the ray starts from the user's virtual hand position, and its direction is determined by the vector from virtual head position to virtual hand position. In order to ensure the teleportation accuracy, different ray lengths were tested in-house before $12 \mathrm{~m}$ is set. Collision detection is performed between the ray and the ground of the virtual environment. Once a collision is detected, the position of collision point will be stored and used as a target position in the next frame.

Inspired by the work of Bozgeyikli et al. [5], the rolling axis of hand is used as an additional control to specify the direction for teleportation. For an ergonomic reason, a linear gain function is applied for mapping limited hand rotation $\left(\theta_{\text {left }}, \theta_{\text {right }}\right)$ to teleportation direction control with 360-degree capability $\left(\theta_{\text {left }}^{\prime}, \theta_{\text {right }}^{\prime}\right)$. The computation is symmetric for left hand and right-hand user.

$$
\begin{gathered}
\theta_{\text {left }}^{\prime}=\theta_{\text {left }} \times 3, \theta_{\text {left }} \in\left[0^{\circ}, 60^{\circ}\right] \\
\theta_{\text {right }}^{\prime}=\theta_{\text {right }} \times 2, \theta_{\text {right }} \in\left[0^{\circ}, 90^{\circ}\right]
\end{gathered}
$$

Teleportation will be activated by pulling the trigger on the Wii controller. Same as Bozgeyikli et al [5], the visual cues used for teleportation consists of a laser beam, a dotted ring lay on the destination position and a 3D arrow above the ring for showing direction. The default color for the whole setup is green. See Fig. 2.

When no collision between the ray and virtual ground is detected (e.g. users choose a position far exceed the length of the ray), the laser beam will be presented as red. Under this circumstance, the user will stay at the same place in the virtual environment even the teleportation is activated.

A cube shape collider surrounds every virtual obstacle in the virtual environment (e.g. walls and tables). User can only be teleported around the obstacle instead of entering into it. During the whole navigation, user can move physically inside the real interaction space which remedy the inevitable accuracy limits from the gesture-based destination selection. To ensure user security, a warning sign will fade into user viewpoint once the user gets too close to the real workspace boundaries. The warning sign consists of a red exclamation mark and a barrier tape following the edges of the physical workspace. With the slanted 


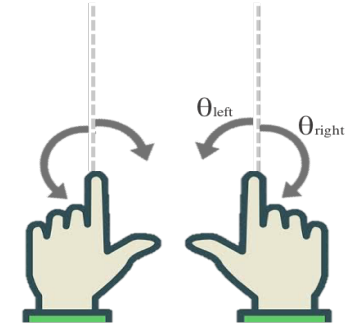

(A)

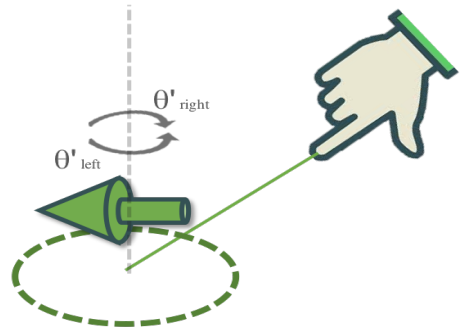

(B)

Fig. 2. (A) Illustrate the rolling axis of hand for teleportation orientation control. (B) Visual presentation for teleportation with direction control

black and tallow stripes texture of the barrier tape, an implicit message "do not cross" is transferred to the user.

\subsection{Virtual environment and the box-opening task}

In the virtual environment, four box sets were located at four similar rooms (A, B, C, and D). These rooms were connected by four corridors with an opposite direction and a center room. A virtual desktop monitor was placed in the middle of the center room.

Each box set composed of four boxes, formed a U-shape and was placed with four different orientations $\left(0^{\circ}, 90^{\circ}, 180^{\circ}, 270^{\circ}\right)$ regarding to the corresponding corridor's direction. The U-shape box setting would map the three screens of the CAVE system after the switch completed, which allowed users to take maximum advantage of physical workspace to complete the task. Each participant needed to navigate to the corresponding room to open successively three of four boxes following a previously defined order. For avoiding the learning effect, two sequences for opening the three boxes were defined. The fourth box was deployed as a distractor and was positioned randomly in the box set. See Fig. 3.

All boxes had a similar shape, but different colour and were named by the room name connecting with a random number (e.g. box B2 located in room B). The label of the box could be seen at each side of the box and was placed with a random orientation. Users thus paid the same cognitive effort to read and understand the box label from either side of the box. By holding the "A" button on the Wii remote controller, a virtual key would be displayed at the user's virtual hand position. Instead, the ray used for teleportation would disappear. The box could be opened from any side whenever a collision was detected between the virtual key and the box.

Participants started the evaluation by standing in front of the virtual monitor where the label of the first box to open was displayed. Participants needed to travel to the corresponding room via the connecting corridor using teleportation technique and opened the target box. The label of the next box to open could 


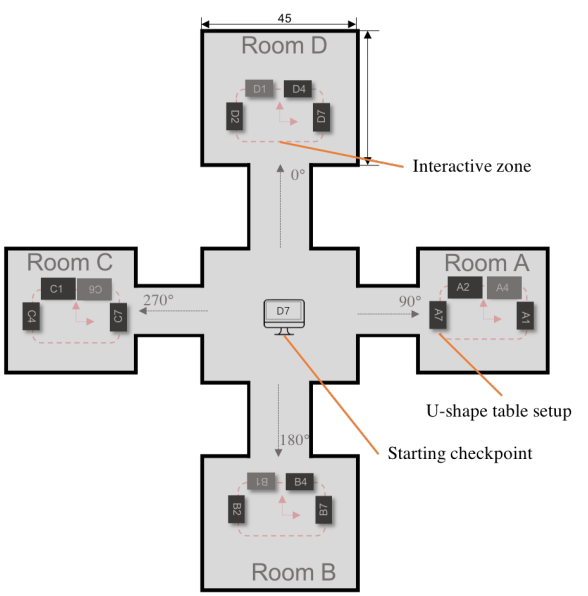

(A)
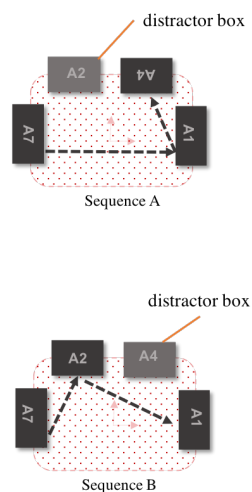

(B)

Fig. 3. (A) Top view of the virtual environment. (B) Illustration of the U-shape box setup and the two sequences defined for opening the box.

be found in the previously opened box. Participants would be guided to the next room when all three boxes were opened in one room. The task repeated eight times and ended when all 4 rooms been visited twice. Different than in without switch condition where users needed to teleport between the boxes, the switch techniques allowed participants to open all three boxes inside one room by walking.

\subsection{Experiment design}

The experiment was designed as within subjects with the independent value of switch technique. Three conditions had been considered: the without switch, the simple switch and the improved switch. The Latin-square design was constructed for counterbalancing the condition and room sequence.

\subsection{Data collection}

For each trail, we logged the time, the number of times of teleportation (user pulled the trigger on the Wii remote controller), the number of times for the user attempts to open the box (user pressed the "A" button on the Wii remote controller), the number of times that warning signs were triggered as well as the user real and virtual head position and orientation. From this data, following measures were extracted:

- Task completion time (TCT): the duration for users to complete the task. The measurement started when participants entered the room and ended when the third box was opened. 
- Manipulation numbers: the sum of the teleportation times and the number of attempts to open the box. This number was computed inside each room and could be considered as a quantitative measurement of cognitive effort paid for completing the task.

- Warning numbers: the number of times that the warning was triggered due to the user stayed too close to the physical workspace boundary. This number was computed inside each room. It was measured since an excessive display of the warning may frustrate users for completing the task and affect the sensation of continuity of the virtual experience.

- Switch time: the time used to switch from free navigation to the spatial consistency situation. The measurement started when users selected an interactive area and ended when they activated the switch.

- Reaction time: the time that the user spent to find and to open the target virtual box after teleporting inside the interactive area. It started from users entered the interactive area and ended when the first target box was opened. The "ability of the user to retain an awareness of her surrounding during and after travel", namely spatial awareness [4] was deployed as an important factor to evaluate our switch approaches. To quantify the spatial awareness, we measured the time that users needed to reorient themselves to a previously seen object in the scene which was suggested in some similar studies $[4,25]$.

- Head rotation: the cumulative sum of user real head rotation movement. The measurement started when users entered the interactive area. It stopped when the first box was opened. In addition to measuring the reaction time, we wanted to investigate how users reorient themselves to a target object by observing their head movement.

\subsection{Hypotheses}

From our assumptions, the following hypotheses were built for this experiment:

- H1:Compared to the without switch, conditions with switch (the simple switch and the improved switch) will result in higher task performance: less $T C T$, less warning numbers and less manipulation numbers.

- H2:Compared to the simple switch, improved switch will provoke less disorientation: less head rotation, less reaction time and less disorientation reported in subjective questionnaires.

- H3:Conceived as a straightforward technique, the simple switch will result in less Switch time compared to the improved switch.

\subsection{Participants}

Eighteen participants ( 7 female, 11 male) aged between 19 and $36(\mu=26.65$, $\sigma=3.88$ ) participated in this experiment. All participants were the right-handed user. None were colour-blind. Participants had none to intermediate knowledge of virtual reality, and half of them had virtual reality experience before. 


\subsection{Procedure}

After participants entered the testing laboratory, they read and signed the formed consent and filled the background information questionnaire. After a short explanation of the system and the objective of this experiment, we helped participants wearing the shutter glasses and the training stage started. Participants went through a 5 minutes training in a similar virtual scene to become familiar with the navigation technique, the switch technique, and the task procedure. Then, the experiment started. After the participants opened the 24 boxes with one condition, they were given a presence survey for evaluating that condition. The experiment ended with an overall survey in which participants needed to rank the three techniques based on usability, disorientation, and preference.

\subsection{Result}

The results presented in this section were considered statistically significant when $\mathrm{p}<0.05$. In all bar plots, error bars show the $95 \%$ confidence intervals $(\mathrm{CI})$. We found the data was not normally distributed in a Shapiro test, and the data variance was not homogeneous in a Levene test. In the following part of this section, we firstly performed non-parametric Friedman test to compare three switch techniques at a global level. Then more detailed analyses were conducted for paired techniques using the Wilcoxon Signed Rank test with Bonferroni correction. All the analyses were performed using Python with scipy.stats library. We registered 432 trials: 3 conditions $\times 4$ rooms $\times 2$ repetitions $\times 18$ participants. To minimize the noise in our data, we averaged the data for each participant in each condition. We run the analysis based on 54 aggregated data set.

$\boldsymbol{T C T}$ Main effects were found on $T C T[\mathrm{~F}(3,54)=29.78, \mathrm{p}<0.0001]$ with without switch (Mean=40.02, $\mathrm{SD}=15.95)$, simple switch (Mean=13.43, $\mathrm{SD}=2.92)$ and improved switch (Mean=15.95, SD=3.20). Then a further Wilcoxon Signed Rank test with Bonferroni correction revealed that the task was significantly longer to achieve for without switch than for simple switch $(\mathrm{p}=0.0006)$ and for improved switch $(\mathrm{p}=0.0006)$. A Wilcoxon Signed Rank test with Bonferroni correction revealed also a main effect on $T C T$ with simple switch less than improved switch $(\mathrm{p}=0.0469)$. See Fig. 4.

Manipulation numbers Main effects on Manipulation numbers $[\mathrm{F}(3,54)=$ 28.8, $\mathrm{p}<0.0001$ ] were revealed in a Friedman test with without switch (Mean = $14.33, S D=5.35)$, simple switch (Mean $=4.79, S D=0.49)$ and improved switch $($ Mean $=5.01, S D=0.77)$. A Wilcoxon Signed Rank with Bonferroni correction detected that without switch induced significant higher Manipulation numbers compared to simple switch $(\mathrm{p}=0.0006)$ and to improved switch $(\mathrm{p}=0.0006)$. No significant difference was found between simple switch and improved switch for manipulation numbers $(\mathrm{p}=0.3985)$. See Fig. 4 . 

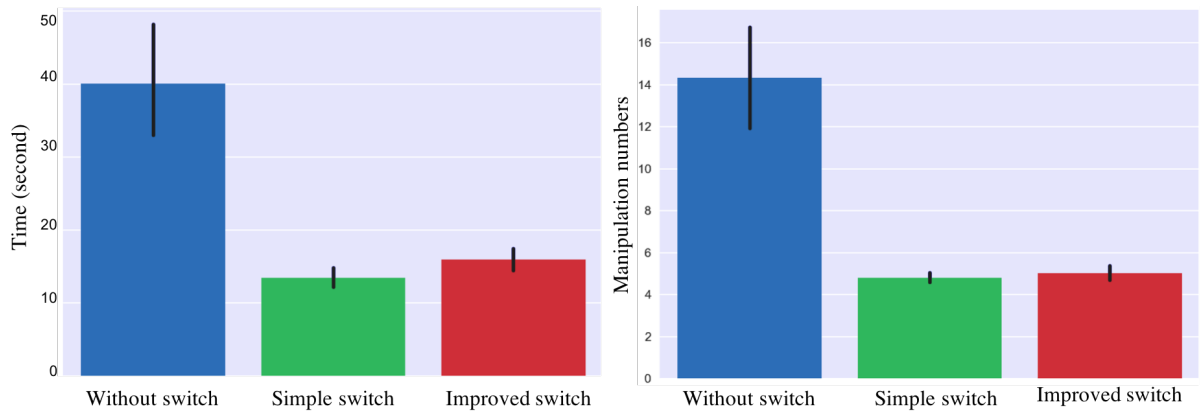

Fig. 4. Bar plots for TCT (left) and manipulation numbers (right)

Warning numbers After a Friedman test, a significant effect $[\mathrm{F}(3,54)=28.59$, $\mathrm{p}<0.0001$ ] on Warning numbers were found with without switch (Mean=1.04, $\mathrm{SD}=0.88)$, simple switch $(\mathrm{Mean}=0.03, \mathrm{SD}=0.07)$ and improved switch Mean $=$ 0.05, SD=0.09). Wilcoxon Signed Rank test with Bonferroni correction indicated that more warning signs were triggered for without switch than for simple switch $(\mathrm{p}=0.0013)$ and improved switch $(\mathrm{p}=0.0013)$. No significant difference was found between simple switch and improved switch for warning numbers $(\mathrm{p}=0.9519)$. See Fig. 5.

Switch time For switch time, a Wilcoxon Signed Rank test with Bonferroni correction revealed that, compared to simple switch (Mean=1.48, $\mathrm{SD}=0.65$ ), improved switch (Mean=4.24, $\mathrm{SD}=1.73$ ) increased significantly the time used for achieving the switch $(\mathrm{p}=0.0006)$. See Fig. 5 .
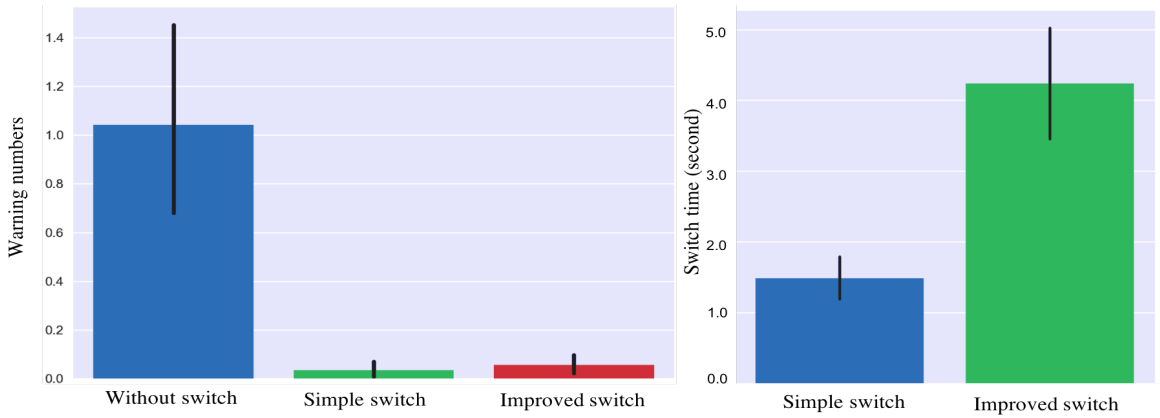

Fig. 5. Bar plots for warning numbers (left) and switch time (right) 
Reaction time Simple switch (Mean $=3.63, \mathrm{SD}=0.82$ ) led to more reaction time compared to improved switch (Mean $=3.30, \mathrm{SD}=0.93$ ), yet the difference was not significant according to a Wilcoxon Signed Rank test with Bonferroni correction $(\mathrm{p}=0.3990)$. See Fig. 6 .

Head rotation Simple switch $($ Mean $=264.84, \mathrm{SD}=108.78)$ produced more head rotation movement compared to improved switch (Mean=207.94, SD=119.40). However, the difference was not significant $(\mathrm{p}=0.5103)$ in a Wilcoxon Signed Rank test with Bonferroni correction. See Fig. 6.
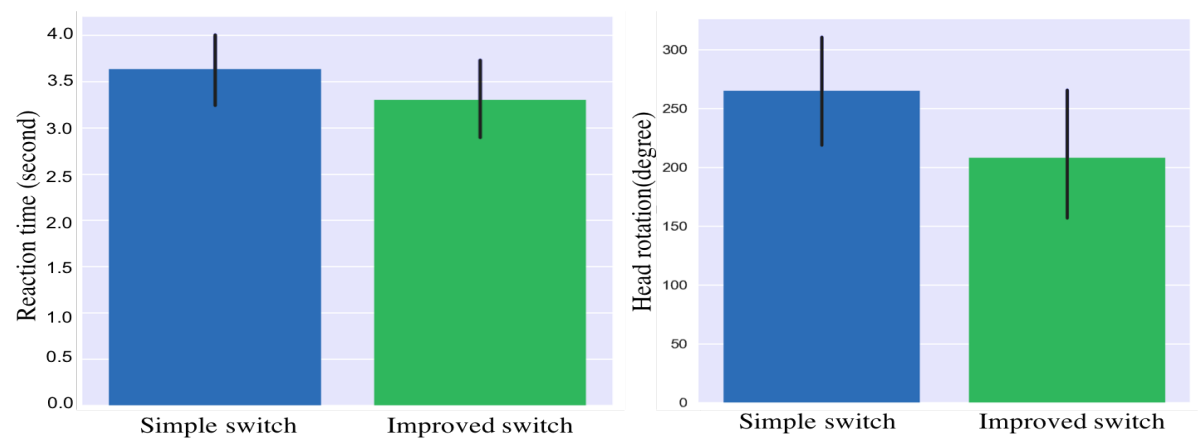

Fig. 6. Bar plots for reaction time(left) and head rotation(right)

Subjective questionnaires A modified version of Witmer and Singer's presence questionnaires [29] was used to measure presence with five levels (0: not at all, 5: completely). Friedman test revealed no significant difference between the three techniques $[\mathrm{F}(3,54)=5.22, \mathrm{p}=0.073]$.

For paired techniques, Wilcoxon Signed Rank test with Bonferroni correction showed that Without switch (Mean $=3.51, \mathrm{SD}=0.54$ ) was perceived less presence than simple switch (Mean $=3.96, \mathrm{SD}=0.42, \mathrm{p}=0.024$ ). No significant presence difference was found between without switch and improved switch (Mean = 3.86, $S D=0.48, p=0.102)$ and between simple switch and improved switch $(\mathrm{p}=0.47)$. See Fig. 7 .

An overall survey was given at the end of all evaluations to asked participants if they found the interaction techniques not mentally demanding and not physically demanding. They also had to rank the three techniques depending on how successful, how disoriented and how frustrated they perceived as well as their preference. Fig. 8 illustrates the result of subjective questionnaires.

At a global level, without switch was more mentally demanding and more physically demanding compared to with switch conditions. It was less preferred by the users. And users perceived more disoriented, more frustrated, and less successful under this condition. See Table 1. 


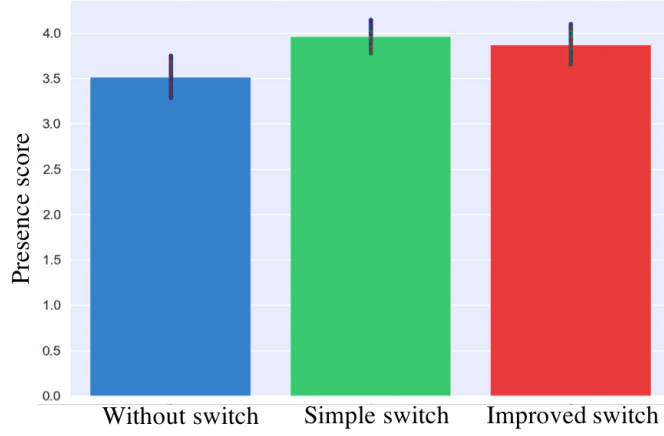

Fig. 7. Bar plots for presence score.

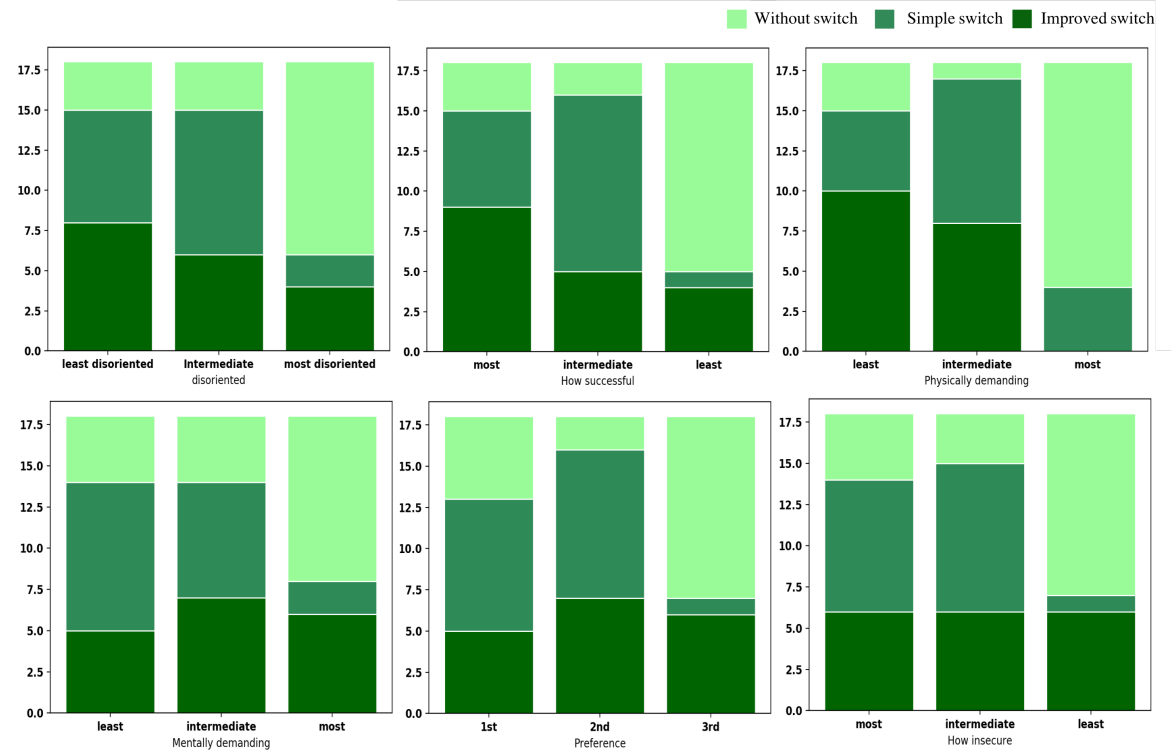

Fig. 8. Stacked percentage bar plot for subjective questionnaire result 
A Wilcoxon Signed Rank test with Bonferroni correction revealed that improved switch was more mentally demanding $(\mathrm{p}=0.0138)$ but less physical demanding $(\mathrm{p}=0.0078)$ compared to the simple switch. Users felt less frustrated with simple switch $(\mathrm{p}=0.0243)$ and it was more favorite by participants $(\mathrm{p}=0.0138)$ compared to the improved switch. No significant difference was revealed for how disoriented and success of the task for these two techniques.

Table 1. Wilcoxon Signed Rank test results for the overall survey.

\begin{tabular}{|l|l|l|}
\hline & Without switch vs. Simple switch & Without switch vs. Improved switch \\
\hline Mentally demanding & Avg.1.66 vs. Avg.2.39 $\mathrm{P}=0.0007$ & Avg.1.66 vs. Avg. 1.94 $\mathrm{P}=0.025$ \\
Physically demanding & Avg.1.38 vs. Avg.2.05 $\mathrm{P}=0.0013$ & Avg.1.38 vs. Avg.2.55 $\mathrm{P}=0.0004$ \\
Disoriented & Avg.1.66 vs. Avg.2.39 $\mathrm{P}=0.0004$ & Avg.1.66 vs. Avg.1.94 $\mathrm{P}=0.0017$ \\
How frustrated & Avg.1.61 vs. Avg. $2.39 \mathrm{P}=0.00046$ & Avg.1.61 vs. Avg. $\mathrm{P}=0.0081$ \\
How successful & Avg.1.44 vs. Avg. $2.28 \mathrm{P}=0.0002$ & Avg.1.44 vs. Avg.2.28 $\mathrm{P}=0.0024$ \\
User preference & Avg.1.5 vs. Avg.2.28 $\mathrm{P}=0.0007$ & Avg. 1.5 vs. Avg.2.22 $\mathrm{P}=0.0253$ \\
\hline
\end{tabular}

\section{Discussion}

\subsection{Without switch compare to with switch}

In general, participants had higher performances while using the two switch techniques for the box-opening task. Both switch conditions reduced the task completion time, the manipulation number, and the warning triggered. This confirmed H1. Besides, compared to the two with switch conditions, without switch was expressed as more mentally and physically demanding in user subjective questionnaire. We interpret this as the spatial consistency between the user physical and virtual workspace ensures the user accessibility to perform the task. In without switch condition, warning signs can be easily triggered in some situations. For example, when there is no virtual obstacle in front, the user will try to walk forward in the virtual environment even the edge of the physical workspace is actually approaching. The warning is thus triggered. More teleportation and time are needed to bridge the distance gap between the user and the target virtual object. The switch helped the user to maintain the spatial consistency in an appropriate area which covered the required virtual interaction spaces to complete the task. As the virtual presentation of user physical workspace was strictly superposed with this area, users can freely walk inside the area without concerning of the real workspace restriction. The user can concentrate on the task and can avoid being distracted by the sudden appearance of warning signs. 


\subsection{Simple switch compare to improved switch}

The improved switch seems provide the user with a better estimate of switch destination and enhance user spatial awareness. With the additional visual feedback of switch destination, users can anticipate the resulting spatial configuration of the switch. By observing the spatial relation between their future destination and the virtual surroundings, users can have more efficient and accurate planning for the following virtual task. Since the relative position between the target virtual object and user future destination is known, the user no longer needs to pay much effort to find the target object after jumping into the interactive area. As a result, users reported the improved switch as less physically demanding. Users also used less reaction time and less head rotation to find the target object with improved switch, although the decreases were not significant compared to Simple switch.

The sensation of disorientation perceived by the user had no significant difference between the two techniques, which is contradicting to what we expected in H2. We interpreted this as the use of u-shape box setup offers user an extra indication of switch destination, which reduces the disorientation perception difference to distinguish these two techniques. The U-shape box setup was designed for using maximum our system to evaluate the impact of user workspace accessibility on task performance. However, it offers users an additional indication of switch destination in the simple switch situation. After several attempts, users can realize that they will be teleported somewhere inside the U-shape setup. And the missing of an accurate indication of teleportation destination seems not to impair that much user spatial awareness.

Significant less switch time was used for simple switch than for improved switch, which validates H3. With the simple switch, user spatial consistency can be recovered quickly and straightforwardly: point the laser beam to a desirable interactive area and activate the switch by pressing the trigger. This confirmed the fact that users considered the simple switch as the least mentally demanding. Although the missing switch destination increased the time used for users reorient themselves to the target object, simple switch still resulted in the least task completion time among three techniques. Since, with improved switch, users spent more time observing and thinking the spatial relation between the switch destination and the nearby virtual contents. Most of the users preferred simple switch than improved switch.

The interface constructs of the switch allow user free to explore the virtual environment and maintain a continual experience to complete some spatial consistency required sub-tasks. With our approach, spatial consistency can be recovered in some specific areas of interest in the virtual environment. The application designers can define these areas corresponding to a complete scenario so that users can visit these areas one by one according to a storyline. Moreover, users can be guided to these areas with a predefined path while active the capability to investigate the nearby features [13] or with a guided avatar [28]. 


\section{Conclusion}

In many virtual reality systems, user real and virtual interaction space are superposed. The spatial consistency allows the user to walk and interact intuitively inside the entire real interaction space, and it enables tangible interface designs. However, user individual capability is usually deactivate in such applications to maintain spatial consistency. In order to provide users with continuous virtual experience in some complex scenarios mixing large scale navigation and spatial consistency required sub-tasks, a seamless switch is necessary to help users recover spatial consistency during virtual navigation.

In this paper, we presented two switch techniques to provide users a smooth transition to recover the spatial consistency during teleportation navigation: simple switch and improved switch. The former is a straightforward technique that allows the user to quickly recover the spatial consistency by two steps: select and activate. The later has additional visual feedback of user switch destination, which dedicates to enhance user spatial awareness and planning accuracy. To verify if the use of switch can ensure user entire physical workspace accessibility, we conducted a user evaluation to compare two switch techniques with a baseline (without switch) in a box-opening task. At a global level, results suggested that the recovering spatial consistency during virtual navigation led to higher task performance compared to the without switch condition. When comparing the two switch techniques, simple switch was faster than improved switch. With an additional visual indication of switch destination, improved switch, seems to provide the user with a better understanding of resulting spatial configuration of switch.

The future study includes extending the switch metaphor in multiple user contexts, in particular when users possess different real interaction space layout. Ideally, users retain identical spatial configurations of real interaction space so that all users' physical workspace can be superimposed to the same virtual area in the same way. But it is not usually the case for distributed or heterogeneous VR systems. Under these circumstances, different layout of real interaction space should be integrated into the shared virtual workspace in some way to ensure user performing a collaborative task. Moreover, presenting the user switch destination by a ghost avatar may help users to have a better understanding of mutual spatial relation, and thus planning an efficient and accuracy switch for all users. Since the switch technique should adapt to the navigation metaphor to maintain a continuous sensation for the whole virtual experience, another subsequent study can be exploring the switch technique for other virtual navigation techniques, e.g., steering navigation.

\section{References}

1. Aguerreche, L., Duval, T., Lécuyer, A.: Comparison of three interactive techniques for collaborative manipulation of objects in virtual reality. In: CGI 2010 (Computer Graphics International) (2010) 
2. Bolte, B., Steinicke, F., Bruder, G.: The jumper metaphor: an effective navigation technique for immersive display setups. In: Proceedings of Virtual Reality International Conference (2011)

3. Bowman, D., Kruijff, E., LaViola Jr, J.J., Poupyrev, I.P.: 3D User interfaces: theory and practice, CourseSmart eTextbook. Addison-Wesley (2004)

4. Bowman, D.A., Koller, D., Hodges, L.F.: Travel in immersive virtual environments: An evaluation of viewpoint motion control techniques. In: Proceedings of IEEE 1997 Annual International Symposium on Virtual Reality. pp. 45-52. IEEE (1997)

5. Bozgeyikli, E., Raij, A., Katkoori, S., Dubey, R.: Point \& teleport locomotion technique for virtual reality. In: Proceedings of the 2016 Annual Symposium on Computer-Human Interaction in Play. pp. 205-216. ACM (2016)

6. Butterworth, J.: 3dm: a three-dimensional modeler using a head-mounted display (1992)

7. Chen, W., Plancoulaine, A., Férey, N., Touraine, D., Nelson, J., Bourdot, P.: 6dof navigation in virtual worlds: comparison of joystick-based and head-controlled paradigms. In: Proceedings of the 19th ACM Symposium on Virtual Reality Software and Technology. pp. 111-114. ACM (2013)

8. Cheng, L.P., Chang, L., Marwecki, S., Baudisch, P.: iturk: Turning passive haptics into active haptics by making users reconfigure props in virtual reality. In: Proceedings of the 2018 CHI Conference on Human Factors in Computing Systems. p. 89. ACM (2018)

9. Cheng, L.P., Marwecki, S., Baudisch, P.: Mutual human actuation. In: Proceedings of the 30th Annual ACM Symposium on User Interface Software and Technology. pp. 797-805. ACM (2017)

10. Cirio, G., Marchal, M., Regia-Corte, T., Lécuyer, A.: The magic barrier tape: a novel metaphor for infinite navigation in virtual worlds with a restricted walking workspace. In: Proceedings of the 16th ACM Symposium on Virtual Reality Software and Technology. pp. 155-162. ACM (2009)

11. Fleury, C., Chauffaut, A., Duval, T., Gouranton, V., Arnaldi, B.: A generic model for embedding users' physical workspaces into multi-scale collaborative virtual environments. In: ICAT 2010 (20th International Conference on Artificial Reality and Telexistence) (2010)

12. Freitag, S., Rausch, D., Kuhlen, T.: Reorientation in virtual environments using interactive portals. In: 2014 IEEE Symposium on 3D User Interfaces (3DUI). pp. 119-122. IEEE (2014)

13. Galyean, T.A.: Guided navigation of virtual environments. In: Proceedings of the 1995 symposium on Interactive 3D graphics. pp. 103-ff. ACM (1995)

14. Hirose, M., Schmalstieg, D., Wingrave, C., Nishimura, K.: Collaborative interaction in co-located two-user scenarios (2009)

15. Hoffman, H.G.: Physically touching virtual objects using tactile augmentation enhances the realism of virtual environments. In: Proceedings. IEEE 1998 Virtual Reality Annual International Symposium (Cat. No. 98CB36180). pp. 59-63. IEEE (1998)

16. Keefe, D.F., Feliz, D.A., Moscovich, T., Laidlaw, D.H., LaViola Jr, J.J.: Cavepainting: a fully immersive $3 \mathrm{~d}$ artistic medium and interactive experience. In: Proceedings of the 2001 symposium on Interactive 3D graphics. pp. 85-93. Citeseer (2001)

17. Reason, J.T., Brand, J.J.: Motion sickness. Academic press (1975)

18. Salzmann, H., Jacobs, J., Froehlich, B.: Collaborative interaction in co-located two-user scenarios. In: Proceedings of the 15th Joint virtual reality Eurographics conference on Virtual Environments. pp. 85-92. Eurographics Association (2009) 
19. Salzmann, H., Moehring, M., Froehlich, B.: Virtual vs. real-world pointing in twouser scenarios. In: Virtual Reality Conference, 2009. VR 2009. IEEE. pp. 127-130. IEEE (2009)

20. Schild, J., Lerner, D., Misztal, S., Luiz, T.: Epicsave - enhancing vocational training for paramedics with multi-user virtual reality. In: 2018 IEEE 6th International Conference on Serious Games and Applications for Health (SeGAH). pp. 1-8. IEEE (2018)

21. Simeone, A.L., Velloso, E., Gellersen, H.: Substitutional reality: Using the physical environment to design virtual reality experiences. In: Proceedings of the 33rd Annual ACM Conference on Human Factors in Computing Systems. pp. 3307-3316. ACM (2015)

22. Simon, A.: First-person experience and usability of co-located interaction in a projection-based virtual environment. In: Proceedings of the ACM symposium on Virtual reality software and technology. pp. 23-30. ACM (2005)

23. Suma, E.A., Lipps, Z., Finkelstein, S., Krum, D.M., Bolas, M.: Impossible spaces: Maximizing natural walking in virtual environments with self-overlapping architecture. IEEE Transactions on Visualization and Computer Graphics 18(4), 555-564 (2012)

24. Suzuki, K., Wakisaka, S., Fujii, N.: Substitutional reality system: a novel experimental platform for experiencing alternative reality. Scientific reports 2, 459 (2012)

25. Tim Weissker, Alexander Kulik, B.F.: Multi-ray jumping: Comprehensible group navigation for collocated users in immersive virtual reality. IEEE (2019)

26. Usoh, M., Arthur, K., Whitton, M.C., Bastos, R., Steed, A., Slater, M., Brooks Jr, F.P.: Walking ¿ walking-in-place $\_$flying, in virtual environments. In: Proceedings of the 26th annual conference on Computer graphics and interactive techniques. pp. 359-364. ACM Press/Addison-Wesley Publishing Co. (1999)

27. Wei $\beta$ ker, T., Kunert, A., Frohlich, B., Kulik, A.: Spatial updating and simulator sickness during steering and jumping in immersive virtual environments. In: 2018 IEEE Conference on Virtual Reality and 3D User Interfaces (VR). pp. 97-104. IEEE (2018)

28. Wernert, E.A., Hanson, A.J.: A framework for assisted exploration with collaboration. In: Proceedings of the conference on Visualization'99: celebrating ten years. pp. 241-248. IEEE Computer Society Press (1999)

29. Witmer, B.G., Singer, M.J.: Measuring presence in virtual environments: A presence questionnaire. Presence 7(3), 225-240 (1998)

30. Xu, M., Murcia-López, M., Steed, A.: Object location memory error in virtual and real environments. In: 2017 IEEE Virtual Reality (VR). pp. 315-316. IEEE (2017) 